\title{
A New Fixed Point Theorem and Applications
}

\author{
Min Fang ${ }^{1}$ and Xie Ping Ding ${ }^{2}$ \\ ${ }^{1}$ Department of Economic Mathematics, South Western University of Finance and Economics, Chengdu, Sichuan 610074, China \\ ${ }^{2}$ College of Mathematics and Software Science, Sichuan Normal University, Chengdu, Sichuan 610068, China
}

Correspondence should be addressed to Xie Ping Ding; xieping_ding@hotmai.com

Received 27 December 2012; Accepted 1 February 2013

Academic Editor: Jen-Chih Yao

Copyright (C) 2013 M. Fang and X. P. Ding. This is an open access article distributed under the Creative Commons Attribution License, which permits unrestricted use, distribution, and reproduction in any medium, provided the original work is properly cited.

A new fixed point theorem is established under the setting of a generalized finitely continuous topological space (GFC-space) without the convexity structure. As applications, a weak KKM theorem and a minimax inequalities of Ky Fan type are also obtained under suitable conditions. Our results are different from known results in the literature.

\section{Introduction}

In the last decade, the theory of fixed points has been investigated by many authors; see, for example, [1-11] and references therein, which has been exploited in the existence study for almost all areas of mathematics, including optimization and applications in economics. Now, there have been a lot of generalizations of the fixed points theorem under different assumptions and different underlying space, and various applications have been given in different fields.

On the other hand, the weak KKM-type theorem introduced by Balaj [12] has attracted an increasing amount of attention and has been applied in many optimization problems so far; see [12-14] and references therein.

Inspired by the research works mentioned above, we establish a collectively fixed points theorem and a fixed point theorem. As applications, a weak KKM theorem and a minimax inequalities of Ky Fan type are also obtained under suitable conditions. Our results are new and different from known results in the literature.

The rest of the paper is organized as follows. In Section 2, we first recall some definitions and theorems. Section 3 is devoted to a new collectively fixed points theorem under noncompact situation on GFC-space and a new fixed point theorem. In Section 4, we show a new weak KKM theorem in underlying GFC-space, and, by using the weak KKM theorem, a new minimax inequality of Ky Fan type is developed.

\section{Preliminaries}

Let $X$ be a topological space and $C, D \subseteq X$. Let int $C$ and int $_{D} C$ denote the interior of $C$ in $X$ and in $D$, respectively. Let $\langle A\rangle$ denote the set of all nonempty finite subsets of a set $A$, and let $\Delta_{n}$ denote the standard $n$-dimensional simplex with vertices $\left\{e_{0}, e_{1}, \ldots, e_{n}\right\}$. Let $X$ and $Y$ be two topological spaces. A mapping $T: X \rightarrow 2^{Y}$ is said to be upper semicontinuous (u.s.c.) (resp., lower semicontinuous (l.s.c)) if for every closed subset $B$ of $Y$, the set $\{x \in X: T(x) \cap B \neq \emptyset\}$ (resp., $\{x \in X$ : $T(x) \subseteq B\})$ is closed.

A subset $A$ of $X$ is said to be compactly open (resp., compactly closed) if for each nonempty compact subset $K$ of $X, A \cap K$ is open (resp., closed) in $K$.

These following notions were introduced by Hai et al. [15].

Definition 1. Let $X$ be a topological space, $Y$ a nonempty set, and $\Phi$ a family of continuous mappings $\varphi: \Delta_{n} \rightarrow X, n \in \mathbf{N}$. A triple $(X, Y, \Phi)$ is said to be a generalized finitely continuous topological space (GFC-space) if and only if for each finite subset $N=\left\{y_{0}, y_{1}, \ldots, y_{n}\right\}$ of $Y$, there is $\varphi_{N}: \Delta_{n} \rightarrow X$ of the family $\Phi$. 
In the sequel, we also use $\left(X, Y,\left\{\varphi_{N}\right\}\right)$ to denote $(X, Y, \Phi)$.

Definition 2. Let $S: Y \rightarrow 2^{X}$ be a multivalued mapping. A subset $D$ of $Y$ is called an $S$-subset of $Y$ if and only if for each $N=\left\{y_{0}, y_{1}, \ldots, y_{n}\right\} \subseteq Y$ and each $\left\{y_{i_{0}}, y_{i_{1}}, \ldots, y_{i_{k}}\right\} \subseteq$ $N \cap D$, one has $\varphi_{N}\left(\Delta_{k}\right) \subset S(D)$, where $\Delta_{k}$ is the face of $\Delta_{n}$ corresponding to $\left\{y_{i_{0}}, y_{i_{1}}, \ldots, y_{i_{k}}\right\}$, that is, the simplex with vertices $\left\{e_{i_{0}}, e_{i_{1}}, \ldots, e_{i_{k}}\right\}$. Roughly speaking, if $D$ is an $S$-subset of $Y$, then $(S(D), D, \Phi)$ is a GFC-space.

The class of GFC-space contains a large number of spaces with various kinds of generalized convexity structures such as FC-space and G-convex space (see [15-17]).

Definition 3 (see [8]). Let $\left(X, Y,\left\{\varphi_{N}\right\}\right)$ be a GFC-space and $Z$ a nonempty set. Let $T: X \rightarrow 2^{Z}$ and $F: Y \rightarrow 2^{Z}$ be two set-valued mappings; $F$ is called a weak KKM mapping with respect to $T$, shortly, weak T-KKM mapping if and only if for each $N=\left\{y_{0}, y_{1}, \ldots, y_{n}\right\} \subseteq Y,\left\{y_{i_{0}}, y_{i_{1}}, \ldots, y_{i_{k}}\right\} \subseteq N$ and $x \in \varphi_{N}\left(\Delta_{k}\right), T(x) \cap \bigcup_{j=0}^{k} F\left(y_{i_{j}}\right) \neq \emptyset$.

Definition 4 (see [8]). Let $X$ be a Hausdorff space, $\left(X, Y,\left\{\varphi_{N}\right\}\right)$ a GFC-space, $Z$ a topological space, $T: X \rightarrow 2^{Z}, f: Y \times Z \rightarrow$ $\mathbf{R} \cup\{-\infty,+\infty\}$, and $g: X \times Z \rightarrow \mathbf{R} \cup\{-\infty,+\infty\}$. Let $\lambda \in \mathbf{R}$. $f$ is called $(\lambda, T, g)$-GFC quasiconvex if and only if for each $x \in X, z \in T(x), N=\left\{y_{0}, y_{1}, \ldots, y_{n}\right\} \in\langle Y\rangle$, and $N_{k}=$ $\left\{y_{i_{0}}, y_{i_{1}}, \ldots, y_{i_{k}}\right\} \subseteq N$, one has the implication $f\left(y_{i_{j}}, z\right)<\lambda$, for all $j=0,1, \ldots, k$ implies that $g\left(x^{\prime}, z\right)<\lambda$ for all $x^{\prime} \in$ $\varphi_{N}\left(\Delta_{k}\right)$

For $\lambda \in \mathbf{R}$, define $\beta \in \mathbf{R}$ and $H_{\lambda}: Y \rightarrow 2^{Z}$ by $\beta=$ $\inf _{x \in X} \sup _{z \in T(x)} g(x, z)$ and $H_{\lambda}(y)=\{z \in Z: f(y, z) \geq \lambda\}$, respectively.

Lemma 5 (see [8]). For $\lambda<\beta$, if $f$ is $(\lambda, T, g)$-GFC quasiconvex, then $H_{\lambda}$ is a weak T-KKM mapping.

The following result is the obvious corollary of Theorem 3.1 of Khanh et al. [8].

Lemma 6. Let $\left\{\left(X_{i}, Y_{i},\left\{\varphi_{N_{i}}\right\}\right)\right\}_{i \in I}$ be a family of GFC-spaces and $X=\prod_{i \in I} X_{i}$ a compact Hausdorff space. For each $i \in I$, let $G_{i}: X \rightarrow 2^{X_{i}}$ and $F_{i}: X \rightarrow 2^{Y_{i}}$ be such that the conditions hold as follows:

(i) for each $x \in X$, each $N_{i}=\left\{y_{0}^{i}, y_{1}^{i}, \ldots, y_{n_{i}}^{i}\right\} \subseteq Y_{i}$ and each $\left\{y_{i_{0}}^{i}, y_{i_{1}}^{i}, \ldots, y_{i_{k_{i}}}^{i}\right\} \subseteq N_{i} \cap F_{i}(x)$, one has $\varphi_{N_{i}}\left(\Delta_{k}\right) \subseteq$ $G_{i}(x)$ for all $i \in I$,

(ii) $X=\bigcup_{y^{i} \in Y_{i}}$ int $F_{i}^{-1}\left(y^{i}\right)$ for all $i \in I$.

Then, there exists $\bar{x}=\left(\bar{x}_{i}\right)_{i \in I} \in X$ such that $\bar{x}_{i} \in G_{i}(\bar{x})$ for all $i \in I$.

\section{Fixed Points Theorems}

Let $I$ be an index set, $X_{i}$ topological spaces, $X=\prod_{i \in I} X_{i}$, and $G_{i}: X \rightarrow 2^{X_{i}}$. The collectively fixed points problem is to find $\bar{x}=\left(\bar{x}_{i}\right)_{i \in I} \in X$ such that $\bar{x}_{i} \in G_{i}(\bar{x})$, for all $i \in I$.
Theorem 7. Let $\left\{\left(X_{i}, Y_{i},\left\{\varphi_{N_{i}}\right\}\right)\right\}_{i \in I}$ be a family of GFC-spaces and $X=\prod_{i \in I} X_{i}$ a Hausdorff space. For each $i \in I$, let $G_{i}$ : $X \rightarrow 2^{X_{i}}, F_{i}: X \rightarrow 2^{Y_{i}}$, and $S_{i}: Y_{i} \rightarrow 2^{X_{i}}$ with the following properties:

(i) for each $x \in X, N_{i}=\left\{y_{0}^{i}, y_{1}^{i}, \ldots, y_{n_{i}}^{i}\right\} \subseteq Y_{i}$, and $\left\{y_{j_{0}}^{i}, y_{j_{1}}^{i}, \ldots, y_{j_{k_{i}}}^{i}\right\} \subseteq N_{i} \cap F_{i}(x)$, one has $\varphi_{N_{i}}\left(\Delta_{k}\right) \subseteq$ $G_{i}(x)$ for all $i \in I$,

(ii) for each compact subset $K$ of $X$ and each $i \in I, K \subseteq$ $\bigcup_{y^{i} \in Y_{i}}$ int $F_{i}^{-1}\left(y^{i}\right)$

(iii) there exists a nonempty compact subset $K_{i}$ of $X_{i}$ and for each $N_{i} \in\left\langle Y_{i}\right\rangle$, there exists an $S_{i}$-subset $L_{N_{i}}$ of $Y_{i}$ containing $N_{i}$ with $S_{i}\left(L_{N_{i}}\right)$ being compact such that

$$
S\left(L_{N}\right) \backslash K \subset \bigcup_{y^{i} \in L_{N_{i}}} \operatorname{int} F_{i}^{-1}\left(y^{i}\right),
$$

where $L_{N}=\prod_{i \in I} L_{N_{i}}, K=\prod_{i \in I} K_{i}$, and $S\left(L_{N}\right)=$ $\prod_{i \in I} S_{i}\left(L_{N_{i}}\right)$. all $i \in I$.

Then, there exists $\bar{x}=\left(\bar{x}_{i}\right)_{i \in I} \in X$ such that $\bar{x}_{i} \in G_{i}(\bar{x})$ for

Proof. As $K$ is a compact subset of $X$, by the condition (ii), there exists a finite set $N_{i}=\left\{y_{0}^{i}, y_{1}^{i}, \ldots, y_{n_{i}}^{i}\right\} \subseteq Y_{i}$, such that

$$
K \subseteq \bigcup_{k=0}^{n_{i}} \operatorname{int} F_{i}^{-1}\left(y_{i_{k}}^{i}\right) \text {. }
$$

By the condition (iii), there exists an $S_{i}$-subset $L_{N_{i}}$ of $Y_{i}$ containing $N_{i}$ such that

$$
S\left(L_{N}\right) \backslash K \subset \bigcup_{y^{i} \in L_{N_{i}}} \operatorname{int} F_{i}^{-1}\left(y^{i}\right),
$$

and it follows that

$$
S\left(L_{N}\right) \subset \bigcup_{y^{i} \in L_{N_{i}}} \operatorname{int} F_{i}^{-1}\left(y^{i}\right) .
$$

We observe that the family $\left.\left\{S_{i}\left(L_{N_{i}}\right), L_{N_{i}},\left\{\varphi_{N_{i}}\right\}\right)\right\}_{i \in I}$ is a family of GFC-space and $S_{i}\left(L_{N_{i}}\right)$ is compact for each $i \in I$, defining set-valued mapping $G_{i}^{*}: S_{i}\left(L_{N_{i}}\right) \rightarrow 2^{S_{i}\left(L_{N_{i}}\right)}$ and $F_{i}^{*}:$ $S_{i}\left(L_{N_{i}}\right) \rightarrow 2^{L_{N_{i}}}$ as follows:

$$
\begin{gathered}
G_{i}^{*}(x)=G_{i}(x) \cap S_{i}\left(L_{N_{i}}\right), \\
F_{i}^{*}(x)=F_{i}(x) \cap L_{N_{i}} .
\end{gathered}
$$

We check assumptions (i) and (ii) of Lemma 6 for replaced $G_{i}$ and $F_{i}$ by $G_{i}^{*}$ and $F_{i}^{*}$, respectively. By (i) and the definition of $S$-subset, for each $x \in S_{i}\left(L_{N_{i}}\right)$, each $N_{i}=\left\{y_{0}^{i}, y_{1}^{i}, \ldots, y_{n_{i}}^{i}\right\} \subseteq$ $L_{N_{i}}$ and each $\left\{y_{j_{0}}^{i}, y_{j_{1}}^{i}, \ldots, y_{j_{k_{i}}}^{i}\right\} \subseteq N_{i} \cap F_{i}^{*}(x)=N_{i} \cap F_{i}(x) \cap L_{N_{i}}$, we have

$$
\varphi_{N_{i}}\left(\Delta_{k}\right) \subseteq G_{i}(x) \cap S_{i}\left(L_{N_{i}}\right)=G_{i}^{*}(x),
$$

then assumption (i) of Lemma 6 is satisfied. 
By (4), we have

$$
\begin{aligned}
S\left(L_{N}\right) & =\left(\bigcup_{y^{i} \in L_{N_{i}}} \operatorname{int} F_{i}^{-1}\left(y^{i}\right)\right) \cap S\left(L_{N}\right) \\
& =\bigcup_{y^{i} \in L_{N_{i}}} \operatorname{int}_{S\left(L_{N}\right)}\left(F_{i}^{-1}\left(y^{i}\right) \cap S\left(L_{N}\right)\right) .
\end{aligned}
$$

On the other hand, for all $y^{i} \in L_{N_{i}}$,

$$
\begin{aligned}
\left(F_{i}^{*}\right)^{-1}\left(y^{i}\right) & =\left\{x \in X: y^{i} \in F_{i}(x)\right\} \cap S\left(L_{N}\right) \\
& =F_{i}^{-1}\left(y^{i}\right) \cap S\left(L_{N}\right) .
\end{aligned}
$$

Hence,

$$
S\left(L_{N}\right)=\bigcup_{y^{i} \in L_{N_{i}}} \operatorname{int}_{S\left(L_{N}\right)}\left(F^{*}\right)^{-1}\left(y^{i}\right) .
$$

Thus, (ii) of Lemma 6 is also satisfied. According to Lemma 6 , there exists a point $\bar{x}=\left(\bar{x}_{i}\right)_{i \in I} \in X$ such that $\bar{x}_{i} \in G_{i}(\bar{x})$ for all $i \in I$.

Remark 8. Theorem 7 generalizes Theorem 3.4 of Ding [6] from FC-space to GFC-space, and our condition (iii) is different from its condition (iii). Theorem 7 also extends Theorem 3 in [18]. Note that Theorem 7 is the variation of Theorem 3.2 in [8].

As a special case of Theorem 7, we have the following fixed point theorem that will be used to prove a weak KKM theorem in Section 4.

Corollary 9. Let $X$ be the Hausdorff space, $\left(X, Y,\left\{\varphi_{N}\right\}\right)$ a GFC-space, $G: X \rightarrow 2^{X}, F: X \rightarrow 2^{Y}$, and $S: Y \rightarrow 2^{X}$ with the following properties:

(i) for each $x \in X, N=\left\{y_{0}, y_{1}, \ldots, y_{n}\right\} \subseteq Y$, and $\left\{y_{i_{0}}, y_{i_{1}}, \ldots, y_{i_{k}}\right\} \subseteq N \cap F(x)$, one has $\varphi_{N}\left(\Delta_{k}\right) \subseteq G(x)$,

(ii) for each compact subset $K$ of $X, K \subseteq \bigcup_{y \in Y}$ int $F^{-1}(y)$,

(iii) for each $N \in\langle Y\rangle$, there exists an S-subset $L_{N}$ of $Y$ containing $N$ with $S\left(L_{N}\right)$ being compact such that

$$
S\left(L_{N}\right) \backslash K \subset \bigcup_{y \in L_{N}} \operatorname{int} F^{-1}(y) .
$$

Then, there exists $\bar{x} \in X$ such that $\bar{x} \in G(\bar{x})$.

\section{Applications}

Theorem 10. Let $X$ be a Hausdorffspace, $\left(X, Y,\left\{\varphi_{N}\right\}\right)$ a GFCspace, $Z$ a nonempty set, $T: X \rightarrow 2^{Z}, H: Y \rightarrow 2^{Z}$, and $S: Y \rightarrow 2^{X}$; assume that

(i) $H$ is a weak T-KKM mapping,

(ii) for each $y \in Y$, the set $\{x \in X: T(x) \cap H(y) \neq \emptyset\}$ is compactly closed, (iii) there exists a compact $K$ of $X$, and, for any $N \in\langle Y\rangle$, there exists an $S$-subset $L_{N}$ of $Y$ containing $N$ with $S\left(L_{N}\right)$ being compact such that

$$
S\left(L_{N}\right) \backslash K \subset \bigcup_{y \in L_{N}} \operatorname{int}\{x \in X: T(x) \cap H(y)=\emptyset\} .
$$

Then, there exists a point $\bar{x} \in X$ such that $T(\bar{x}) \cap H(y) \neq \emptyset$ for each $y \in Y$.

Proof. Define $F: X \rightarrow 2^{Y}$ and $G: X \rightarrow 2^{X}$ by

$$
\begin{gathered}
F(x)=\{y \in Y: T(x) \cap H(y)=\emptyset\}, \\
G(x)=\left\{x^{\prime} \in X: \exists y \in F(x), T\left(x^{\prime}\right) \cap H(y) \neq \emptyset\right\} .
\end{gathered}
$$

Suppose the conclusion does not hold. Then, for each $x \in$ $X$, there exists a $y \in Y$ such that

$$
T(x) \cap H(y)=\emptyset .
$$

It is easy to see that $F$ has nonempty values. By (ii), for each $y \in Y$,

$$
F^{-1}(y)=\{x \in X: T(x) \cap H(y)=\emptyset\}
$$

is compactly open. Then,

$$
X=\bigcup_{y \in Y} \operatorname{int} F^{-1}(y) .
$$

Since $K$ is a compact subset of $X$, then there exists $N \in\langle Y\rangle$ such that

$$
K \subseteq \bigcup_{y \in N} \operatorname{int} F^{-1}(y)
$$

Then, assumption (ii) of Corollary 9 is satisfied.

It follows from (iii) that there exists a compact $K$ of $X$ and for any $N \in\langle Y\rangle$, there exists a $S$-subset $L_{N}$ of $Y$ containing $N$ with $S\left(L_{N}\right)$ being compact such that

$$
S\left(L_{N}\right) \backslash K \subset \bigcup_{y \in L_{N}} \operatorname{int} F^{-1}(y) .
$$

Therefore, assumption (iii) of Corollary 9 is also satisfied.

Furthermore, $G$ has no fixed point. Indeed, if $x \in G(x)$, then there exists $y \in F(x)$ such that

$$
T(x) \cap H(y) \neq \emptyset,
$$

which contracts the definition of $F$. Thus, assumption (i) of Corollary 9 must be violated; that is, there exist an $\bar{x} \in X$, $\bar{N}=\left\{\bar{y}_{0}, \bar{y}_{1}, \ldots, \bar{y}_{n}\right\} \subseteq Y$, and

$$
\bar{N}_{k}=\left\{\bar{y}_{i_{0}}, \bar{y}_{i_{1}}, \ldots, \bar{y}_{i_{k}}\right\} \subseteq \bar{N} \cap F(\bar{x})
$$

such that

$$
\varphi_{\bar{N}}\left(\Delta_{k}\right) \nsubseteq G(\bar{x}) .
$$


That is, for each $y \in F(\bar{x})$,

$$
T(\bar{x}) \cap H(y)=\emptyset .
$$

Hence,

$$
T(\bar{x}) \cap H\left(\bar{N}_{k}\right)=\emptyset .
$$

On the other hand, since $H$ is a weak T-KKM mapping and $\bar{x} \in \varphi_{\bar{N}}\left(\Delta_{k}\right)$, we have

$$
T(\bar{x}) \cap H\left(\bar{N}_{k}\right) \neq \emptyset,
$$

which is contradict. This completes the proof.

Remark 11. (1) Theorem 10 extends Theorem 1 in [13] from the G-convex space to GFC-space, and our proof techniques are different. Theorem 10 also generalizes Theorem 4.1 of [8] from the compactness assumption to noncompact situation.

(2) If $Z$ is a topological space, condition (ii) in Theorem 10 is fulfilled in any of the following cases (see [13]):

(i) $H$ has closed values, and $T$ is u.s.c, on each compact subset of $X$.

(ii) $H$ has compactly closed values, and $T$ is u.s.c, on each compact of subset of $X$ and its values are compact.

Theorem 12. Let $X$ be a Hausdorff space, $\left(X, Y,\left\{\varphi_{N}\right\}\right)$ a GFCspace, $Z$ a topological space, $T: X \rightarrow 2^{Z}$ u.s.c., $f: Y \times Z \rightarrow$ $\mathbf{R} \cup\{-\infty,+\infty\}$. and $S: Y \rightarrow 2^{X}$; assume that

(i) for each $y \in Y, f(y, \cdot)$ is u.s.c. on each compact subset of $Z$,

(ii) $f$ is $(\lambda, T, g)$-GFC quasiconvex for all $\lambda<\beta$ sufficiently close to $\beta$,

(iii) there exists a compact $K$ of $X$, and, for any $N \in\langle Y\rangle$, there exists an $S$-subset $L_{N}$ of $Y$ containing $N$ with $S\left(L_{N}\right)$ being compact such that

$$
S\left(L_{N}\right) \backslash K \subset \bigcup_{y \in L_{N}} \operatorname{int}\left\{x \in X: T(x) \cap H_{\lambda}(y)=\emptyset\right\} .
$$

Then,

$$
\inf _{x \in X_{z \in T(x)}} \sup _{z} g(x, z) \leq \sup _{x \in X} \inf _{y \in Y} \sup _{z \in T(x)} f(y, z) .
$$

Proof. Let $\lambda<\beta$ be arbitrary. By Lemma 5 and condition (ii), $H_{\lambda}$ is a weak T-KKM mapping. It follows from condition (i) that $H_{\lambda}$ has closed values. Hence, the set $\{x \in X: T(x) \cap$ $\left.H_{\lambda}(y) \neq \emptyset\right\}$ is compactly closed for all $y \in Y$ (see Remark 11 (2)). Thus, all the conditions of Theorem 10 are satisfied, and so there exists an $\bar{x} \in X$ such that

$$
T(\bar{x}) \cap H_{\lambda}(y) \neq \emptyset, \quad \forall y \in Y .
$$

This implies that $\lambda \leq \inf _{y \in Y} \sup _{z \in T(\bar{x})} f(y, z)$ and so

$$
\lambda \leq \sup _{x \in X} \inf _{y \in Y} \sup _{z \in T(\bar{x})} f(y, z) .
$$

Since $\lambda<\beta$ is arbitrary, we get the conclusion. This completes the proof.
Remark 13. Theorem 12 improves Theorem 4.2 of [8] from the compactness assumption to noncompact situation. Theorem 12 also extends Theorem 4 of [12] from compact Gconvex space to noncompact GFC-space. Our result includes corresponding earlier Fan-type minimax inequalities due to Tan [19], Park [20], Liu [21], and Kim [22].

\section{Acknowledgment}

This work was supported by the University Research Foundation (JBK120926).

\section{References}

[1] E. Tarafdar, "A fixed point theorem and equilibrium point of an abstract economy," Journal of Mathematical Economics, vol. 20, no. 2, pp. 211-218, 1991.

[2] K. Q. Lan and J. Webb, "New fixed point theorems for a family of mappings and applications to problems on sets with convex sections," Proceedings of the American Mathematical Society, vol. 126, no. 4, pp. 1127-1132, 1998.

[3] Q. H. Ansari and J.-C. Yao, "A fixed point theorem and its applications to a system of variational inequalities," Bulletin of the Australian Mathematical Society, vol. 59, no. 3, pp. 433-442, 1999.

[4] Q. H. Ansari, A. Idzik, and J.-C. Yao, "Coincidence and fixed point theorems with applications," Topological Methods in Nonlinear Analysis, vol. 15, no. 1, pp. 191-202, 2000.

[5] X. P. Ding and K.-K. Tan, "Fixed point theorems and equilibria of noncompact generalized games," in Fixed Point Theory and Applications, pp. 80-96, World Sicence, Singapore, 1992.

[6] X. P. Ding, "Continuous selection, collectively fixed points and system of coincidence theorems in product topological spaces," Acta Mathematica Sinica, vol. 22, no. 6, pp. 1629-1638, 2006.

[7] L.-J. Lin and Q. H. Ansari, "Collective fixed points and maximal elements with applications to abstract economies," Journal of Mathematical Analysis and Applications, vol. 296, no. 2, pp. 455472, 2004.

[8] P. Q. Khanh, V. S. T. Long, and N. H. Quan, "Continuous selections, collectively fixed points and weak KnasterKuratowski-Mazurkiewicz mappings in optimization," Journal of Optimization Theory and Applications, vol. 151, no. 3, pp. 552$572,2011$.

[9] X. P. Ding and L. Wang, "Fixed points, minimax inequalities and equilibria of noncompact abstract economies in FC-spaces," Nonlinear Analysis A, vol. 69, no. 2, pp. 730-746, 2008.

[10] X. P. Ding, "Collective fixed points, generalized games and systems of generalized quasi-variational inclusion problems in topological spaces," Nonlinear Analysis A, vol. 73, no. 6, pp. 1834-1841, 2010.

[11] G. X.-Z. Yuan, KKM Theory and Applications in Nonlinear Analysis, vol. 218, Marcel Dekker, New York, NY, USA, 1999.

[12] M. Balaj, "Weakly G-KKM mappings, G-KKM property, and minimax inequalities," Journal of Mathematical Analysis and Applications, vol. 294, no. 1, pp. 237-245, 2004.

[13] M. Balaj and D. O'Regan, "Weak-equilibrium problems in Gconvex spaces," Rendiconti del Circolo Matematico di Palermo, vol. 57, no. 1, pp. 103-117, 2008.

[14] G.-S. Tang, Q.-B. Zhang, and C.-Z. Cheng, "W-G-F-KKM mapping, intersection theorems and minimax inequalities in 
FC-space," Journal of Mathematical Analysis and Applications, vol. 334, no. 2, pp. 1481-1491, 2007.

[15] N. X. Hai, P. Q. Khanh, and N. H. Quan, "Some existence theorems in nonlinear analysis for mappings on GFC-spaces and applications," Nonlinear Analysis A, vol. 71, no. 12, pp. 61706181, 2009.

[16] P. Q. Khanh and N. H. Quan, "Intersection theorems, coincidence theorems and maximal-element theorems in GFCspaces," Optimization, vol. 59, no. 1, pp. 115-124, 2010.

[17] P. Q. Khanh and N. H. Quan, "General existence theorems, alternative theorems and applications to minimax problems," Nonlinear Analysis, vol. 72, no. 5, pp. 2706-2715, 2010.

[18] S. Park, "Continuous selection theorems in generalized convex spaces," Numerical Functional Analysis and Optimization, vol. 20, no. 5-6, pp. 567-583, 1999.

[19] K.-K. Tan, "Comparison theorems on minimax inequalities, variational inequalities, and fixed point theorems," Journal of the London Mathematical Society, vol. 28, no. 3, pp. 555-562, 1983.

[20] S. Park, "Generalized Fan-Browder fixed point theorems and their applications," in Collection of Papers Dedicated to J. G. Park, pp. 51-77, 1989.

[21] F.-C. Liu, "On a form of KKM principle and Sup Inf Sup inequalities of von Neumann and of Ky Fan type," Journal of Mathematical Analysis and Applications, vol. 155, no. 2, pp. 420436, 1991.

[22] I. Kim, "KKM theorem and minimax inequalities in G-convex spaces," Nonlinear Analysis Forum, vol. 6, no. 1, pp. 135-142, 2001. 


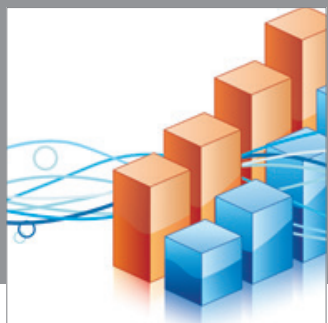

Advances in

Operations Research

mansans

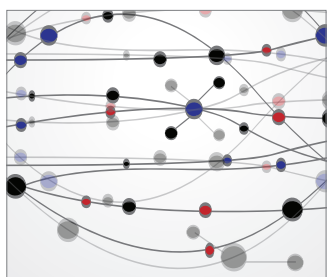

The Scientific World Journal
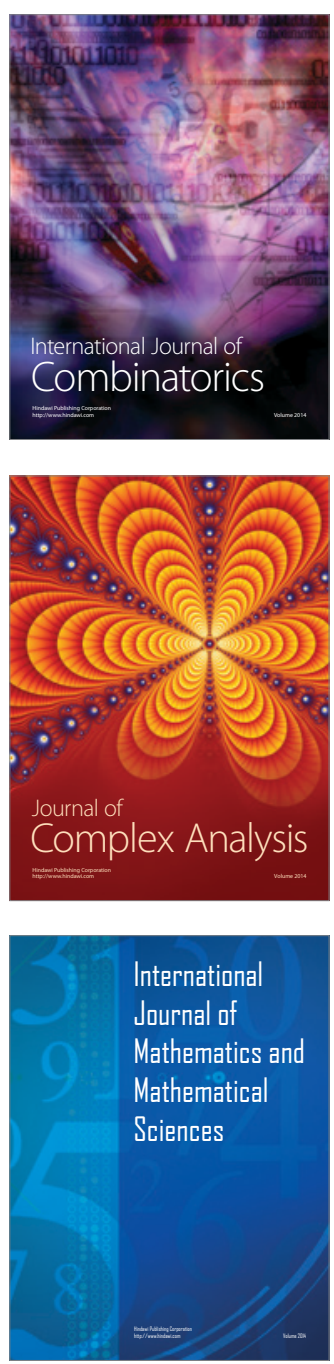
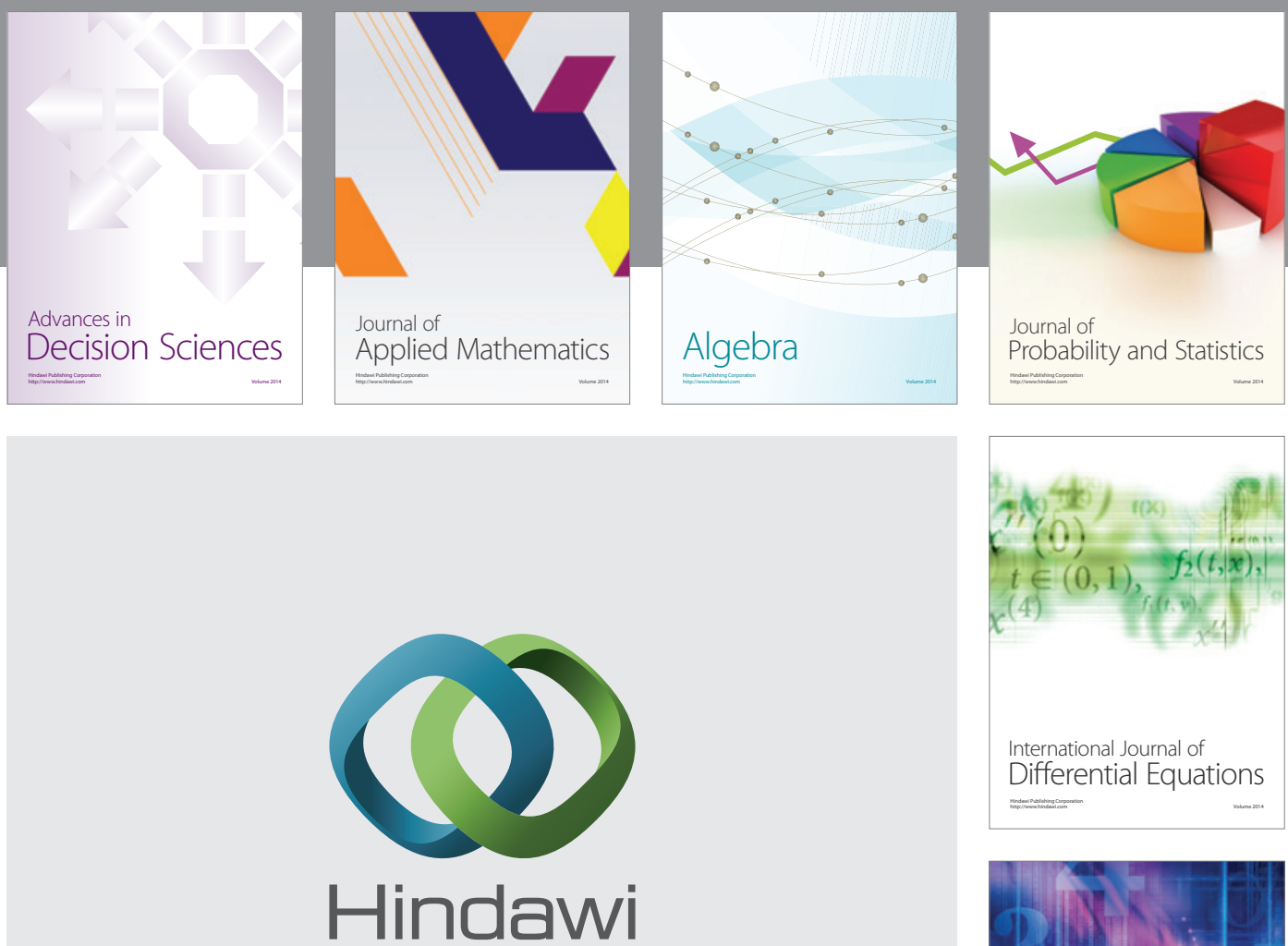

Submit your manuscripts at http://www.hindawi.com
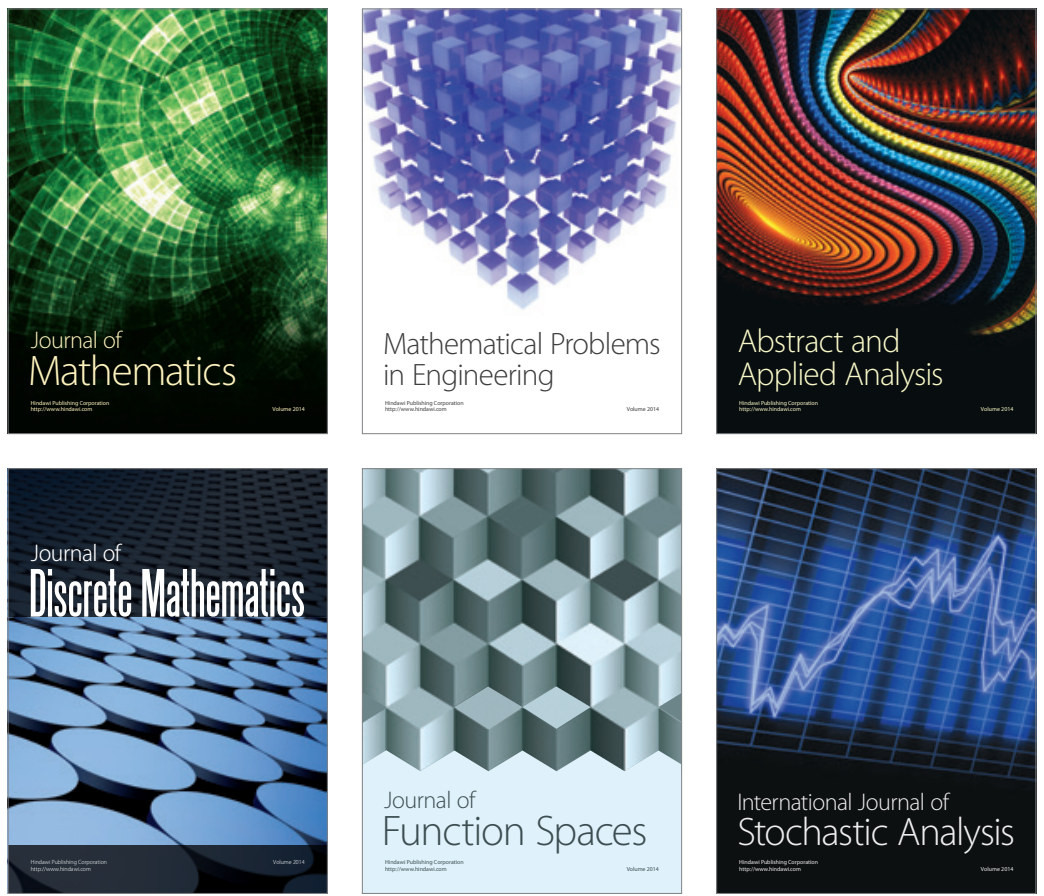

Journal of

Function Spaces

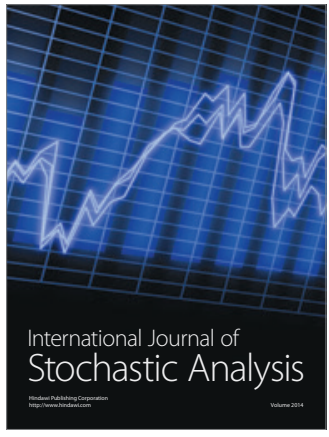

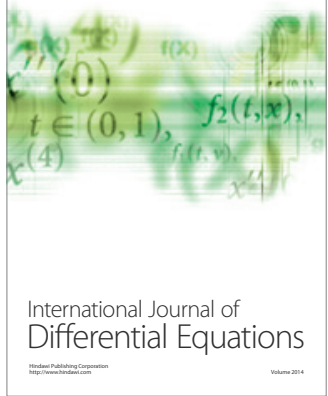
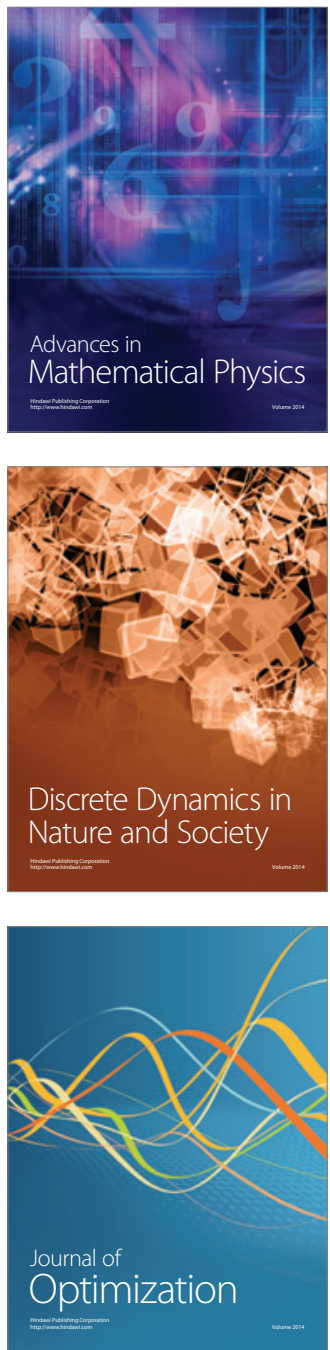